\title{
Antimicrobial effect of topical local anesthetic spray on oral microflora
}

\author{
Ratchapin L Srisatjaluk', Boworn Klongnoi ${ }^{2}$, Natthamet Wongsirichat ${ }^{2}$ \\ Department of Oral Microbiology Faculty of Dentistry Mahidol University, ${ }^{2}$ Department Oral \& Maxillofacial Surgery, Faculty of Dentistry \\ Mahidol University, Bangkok, Thailand
}

\begin{abstract}
Background: To evaluate the antimicrobial activity of lidocaine (LD) topical anesthetic spray against oral microflora. Methods: Antimicrobial effects of 10\% LD spray were assessed against six bacterial cultures obtained from volunteers: Escherichia coli, Enterococcus faecalis, Staphylococcus aureus, Streptococcus salivarius, Streptococcus pyogenes, and Streptococcus sanguinis. The filter papers contained $50-\mu \mathrm{LD}$, brain heart infusion (BHI) broth, or $0.2 \%$ chlorhexidine. Papers were placed on the cultured blood plates for 1-3 min. After the papers were removed, plates were incubated for $24 \mathrm{~h}$. Bacterial growth on the contact areas was recorded as the antimicrobial score. The split mouth technique was use in for sample collection in clinical study. Filter papers soaked with either BHI broth or LD were placed on the right or left buccal mucosa for $1 \mathrm{~min}$, and replaced with other papers to imprint biofilms onto the contact areas. Papers were placed on blood plates, incubated for $24 \mathrm{~h}$, and antimicrobial scores were determined. Experiments were conducted for 2- and 3-min exposure times with a 1-day washout period.

Results: LD exhibited bactericidal effects against E. coli, S. sanguinis, and S. salivarius within 1 min but displayed no effect against $S$. aureus, E. faecalis, and $S$. pyogenes. The antimicrobial effect of LD on oral microflora depended upon exposure time, similar to the results obtained from the clinical study $(\mathrm{P}<0.05)$. LD showed 60-95\% biofilm reduction on buccal mucosa.

Conclusions: Antimicrobial activity of 10\% LD topical anesthetic spray was increased by exposure time. The

3 min application reduced oral microflora in the buccal mucosa.
\end{abstract}

Key Words: Antimicrobial effect; Lidocaine; Local anesthetic; Oral microflora.

\section{INTRODUCTION}

In dentistry, local anesthesia is necessary, especially in the field of oral and maxillofacial surgery. The mechanism of local anesthetics inhibits the transmission of nerve impulses causing temporary loss of sensation at the peripheral organ supplied by the nerve until the anesthetic effect is terminated.

Local anesthetics used in dentistry are divided by structure into 3 classes: ester type, amide type, and saxitoxin and tetrodotoxin type. LD hydrochloride is an amide anesthetic that was developed for use in clinical practice to replace ester anesthetics that were associated with a higher incidence of allergic reactions due to one of their metabolites, para-amino benzoic acid (PABA).

LD has a rapid onset, moderate duration of action, and fewer complications, which facilitate its current widespread use. In clinical practice, LD is commonly used for injection at a $2 \%$ concentration. At higher concentrations, it can be used as a topical local anesthetic. Besides pain blocking properties, antimicrobial effects of several local anesthetics containing LD have been reported [1-7]. Divergent results are due to the drug concentration, type of microorganism tested, method of study and various additives, such as preservatives or
Copyright(C) 2016 Journal of Dental Anesthesia and Pain Medicine
Received: 2016. March. 6. - Revised: 2016. March. 10. - Accepted: 2016. March. 16 Corresponding Author: Natthamet Wongsirichat, Oral Maxillofacial Surgery, Department Faculty of Dentistry, Mahidol University, 6 Yothi Street Rachathewee District Bangkok 10400 Thailand

Tel: +66-22007777, ext 3333 E-mail: natthamet.won@mahidol.ac.th 
vasoconstrictive components such as epinephrine [7-8]. Topical local anesthetics are commonly used for application on sensitive areas for the purpose of sample collection, e.g., the cornea or the upper respiratory tract, or site of needle injection to reduce the pain. Antimicrobial properties of topical local anesthetics have been reported [2,3,5,7,9-13]. In a dental setting, oral injection of local anesthetics may cause tissue damage to some extent at the injection site, thereby increasing the chance of bacteremia due to numerous oral microflora [14].

The use of an antiseptic agent prior to oral injection is still controversial. Instead of using an antiseptic agent to wipe the injection site, most dentists use the topical local anesthetic to block the pain from needle injection. This regimen is supported by the previous in vitro studies that topical anesthetic agents have shown antimicrobial effects and suggested a beneficial role in the prophylaxis of infection $[5,8,13,15]$. In addition, the local infection or abscess at the site of injection is considered exceptional.

Because oral mucosa is commonly colonized with mixed microbial species as biofilms, the clinical antimicrobial effectiveness may vary depending upon the concentration and type of microorganisms. It is thus important to be aware of the antimicrobial effectiveness in clinical settings. The purpose of this study was to evaluate the antimicrobial effectiveness of $10 \% \mathrm{LD}$ topical anesthetic spray against oral microflora in both laboratory and clinical settings.

\section{MATERIALS AND METHODS}

\section{Volunteers for collection of oral microflora and clinical study}

Twenty-two volunteers were recruited for this study. The inclusion criteria included healthy people with same number of teeth on the left and right side of the mouth and the ability to open the mouth for more than $3 \mathrm{~min}$. The exclusion criteria included those with oral lesions on the buccal mucosa, allergies to local anesthesia or chlor- hexidine (CHX), the routine use of antibacterial mouthwash and those that received antibiotics within 2 weeks prior to the study.

The study protocol was approved by the Committee for Human Research, the faculty of Dentistry, and the faculty of Pharmacy at Mahidol University (MU-DT/PYIRB 2014/DT098). The oral microflora was collected from both sides of the buccal mucosa of volunteers using sterile cotton swabs, placed in 2-ml BHI broth, and immediately sent to the microbiology laboratory.

\section{In vitro test for antimicrobial effect}

In all, the following 6 bacterial strains from the stock cultures of the Oral Microbiology Department, Faculty of Dentistry, Mahidol University, were used to determine the antimicrobial effect: Escherichia coli ATCC 25922, Enterococcus faecalis ATCC 9433, Staphylococcus aureus ATCC 6538, Streptococcus salivarius ATCC 19258, Streptococcus pyogenes ATCC 19615, and Streptococcus sanguinis ATCC 10556. Overnight cultures in brain heart infusion (BHI) broth and oral microflora samples from the volunteers were adjusted to the turbidity of the McFarland standard 0.5 and diluted to $10^{3}$ to obtain approximately $1.0 \times 10^{5} \mathrm{CFU} / \mathrm{ml}$. A $100-\mu \mathrm{l}$ aliquot of bacterial solution was plated on three blood agar plates each. The plate was divided into four quadrants each of which was covered by a filter paper containing a test solution.

The test filter papers $\left(2 \times 2 \mathrm{~cm}^{2}\right)$ were prepared by suspension in $50 \mu \mathrm{l}$ of the BHI broth, $0.2 \%$ CHX (Faculty of Dentistry, Mahidol University), or 10\% LD. After filter papers were air-dried for $10 \mathrm{~min}$, they were placed on the surface of each blood agar quadrant. After $1 \mathrm{~min}$, the papers were removed and the plate was incubated at $37^{\circ} \mathrm{C}$ in a $\mathrm{CO}_{2}$ incubator for $24 \mathrm{~h}$. The growth of the bacteria on the LD quadrant was observed and compared to the control quadrants.

The BHI and CHX quadrants served as the negative (bacterial growth) and positive (no bacterial growth) controls, respectively. The antimicrobial effects at 2- and 3-min exposure times were evaluated in similar pro- 
cedures. Bacterial growth on the LD quadrant was presented as the antimicrobial scores shown in Table 1. Each experiment was repeated twice to confirm the antimicrobial score.

\section{In vivo antimicrobial effectiveness}

Antimicrobial effectiveness of $10 \%$ LD local spray against oral microflora was assessed in vivo using the split mouth technique. Each volunteer was evaluated in 4 experiments with varying $\mathrm{LD}$ exposure times of 1,2 , and $3 \mathrm{~min}$

Prior to the experiment, the volunteers rinsed their mouths with sterile normal saline. The filter papers (2 $\times 2 \mathrm{~cm}^{2}$ ) containing BHI broth or $10 \% \mathrm{LD}$ were placed on either side of buccal mucosa above the occlusal plane between the permanent mandibular second premolar and the permanent mandibular first molar for $1 \mathrm{~min}$. Then the paper was replaced by the filter paper with BHI broth, size $1.5 \times 1.5 \mathrm{~cm}^{2}$ for $2 \mathrm{~min}$. The papers were placed on a blood agar plate on the control side (BHI) and the test area (LD) for $2 \mathrm{~min}$.

The plate was incubated at $37^{\circ} \mathrm{C}$ in the $\mathrm{CO}_{2}$ incubator for $24 \mathrm{~h}$. The density of the bacterial growth in the test area was compared with the control area and recorded as antimicrobial scores (0-4), indicated in Table 1. After the washout period of at least 1 day, the volunteers were asked to conduct the experiment at 2 and $3 \mathrm{~min}$ exposure times using LD. In addition, antimicrobial effectiveness of $0.2 \% \mathrm{CHX}$ was also assessed at the exposure time of $2 \mathrm{~min}$

\section{Statistical analysis}

The mean values of antimicrobial scores obtained from 22 volunteers at different exposure times (1, 2, and $3 \mathrm{~min})$ were analyzed by the Friedman test using SPSS version 18.0 statistical package for Windows (SPSS Inc. Chicago, IL). The significance level was considered at $\mathrm{P}<0.05$.

\section{RESULTS}

\section{In vitro test for antimicrobial effect of $10 \%$ LD topical local anesthesia}

The antimicrobial effect of $0.2 \% \mathrm{CHX}$ and $10 \% \mathrm{LD}$ against six bacterial species was shown in Table 2. The in vitro results indicated that CHX could inhibit E. coli, S. salivarius, $S$. viridans, and $S$. aureus, within $1 \mathrm{~min}$ (> 95\% reduction), but it had no effect against E. faecalis

Table 1. Antimicrobial scores of $10 \%$ lidocaine spray by comparing with the growth of negative control (BHI)

\begin{tabular}{lc}
\hline \multicolumn{1}{c}{ Growth compared to the negative control } & Antimicrobial scores \\
\hline No growth or $>95 \%$ bacterial reduction & 4 \\
Growth density was $60-95 \%$ bacterial reduction & 3 \\
Growth density was 40-60\% bacterial reduction & 2 \\
Growth density was 5-40\% bacterial reduction & 1 \\
Growth density comparable to the negative control or less than 5\% bacterial reduction & 0 \\
\hline
\end{tabular}

Table 2. Antimicrobial effect of $0.2 \%$ chlorhexidine and $10 \%$ lidocaine against 6 oral bacterial species

\begin{tabular}{|c|c|c|c|c|c|c|}
\hline \multirow{3}{*}{ Bacterial species } & \multicolumn{6}{|c|}{ Antimicrobial score } \\
\hline & \multicolumn{3}{|c|}{$\begin{array}{l}0.2 \% \text { chlorhexidine } \\
\text { Exposure time (min) }\end{array}$} & \multicolumn{3}{|c|}{$\begin{array}{c}10 \% \text { lidocaine } \\
\text { Exposure time (min) }\end{array}$} \\
\hline & 1 & 2 & 3 & 1 & 2 & 3 \\
\hline E. coli & 4 & 4 & 4 & 3 & 3 & 3 \\
\hline S. viridans & 4 & 4 & 4 & 3 & 3 & 3 \\
\hline S. salivarius & 4 & 4 & 4 & 1 & 1 & 1 \\
\hline S. aureus & 4 & 4 & 4 & 0 & 0 & 0 \\
\hline E. faecalis & 0 & 0 & 0 & 0 & 0 & 0 \\
\hline S. pyogenes & 0 & 0 & 0 & 0 & 0 & 0 \\
\hline
\end{tabular}

Antimicrobial score: $4=>95 \%$ bacterial reduction, $3=60-95 \%$ bacterial reduction, $2=40-60 \%$ bacterial reduction, $1=5-40 \%$ bacterial reduction, $0=<5 \%$ bacterial reduction 


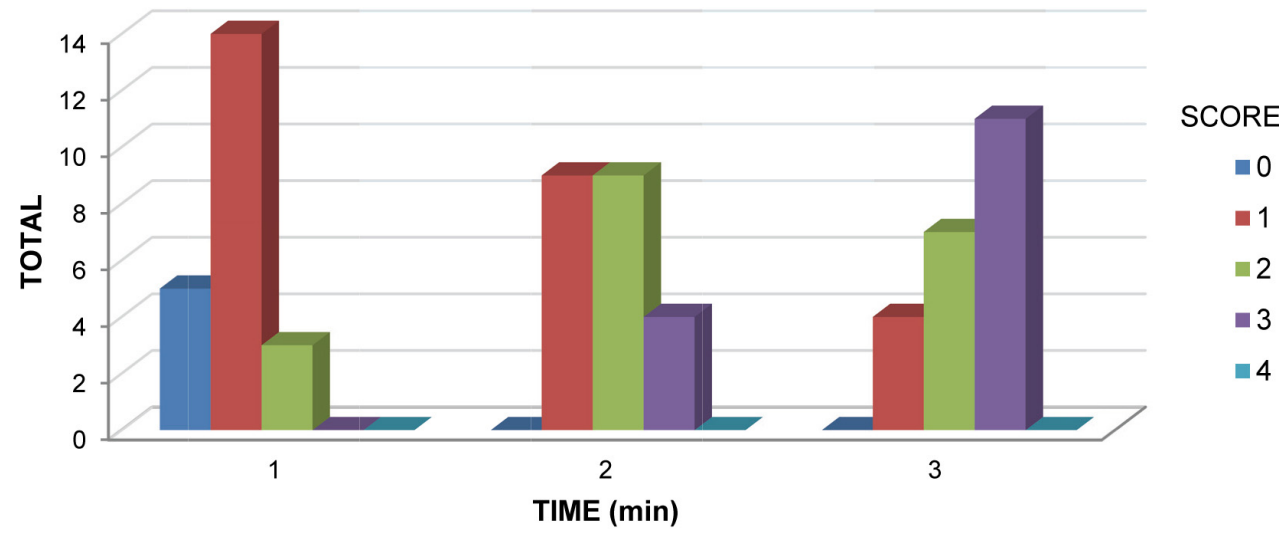

Fig. 1. Antimicrobial effectiveness of $10 \%$ lidocaine against oral microflora collected from the buccal mucosa of 22 volunteers; exposure times of 1, 2, and $3 \mathrm{~min}$. Antimicrobial score: $4=>95 \%$ bacterial reduction, $3=60-95 \%$ bacterial reduction, $2=40-60 \%$ bacterial reduction, $1=5-40 \%$ bacterial reduction, $0=<5 \%$ bacterial reduction.

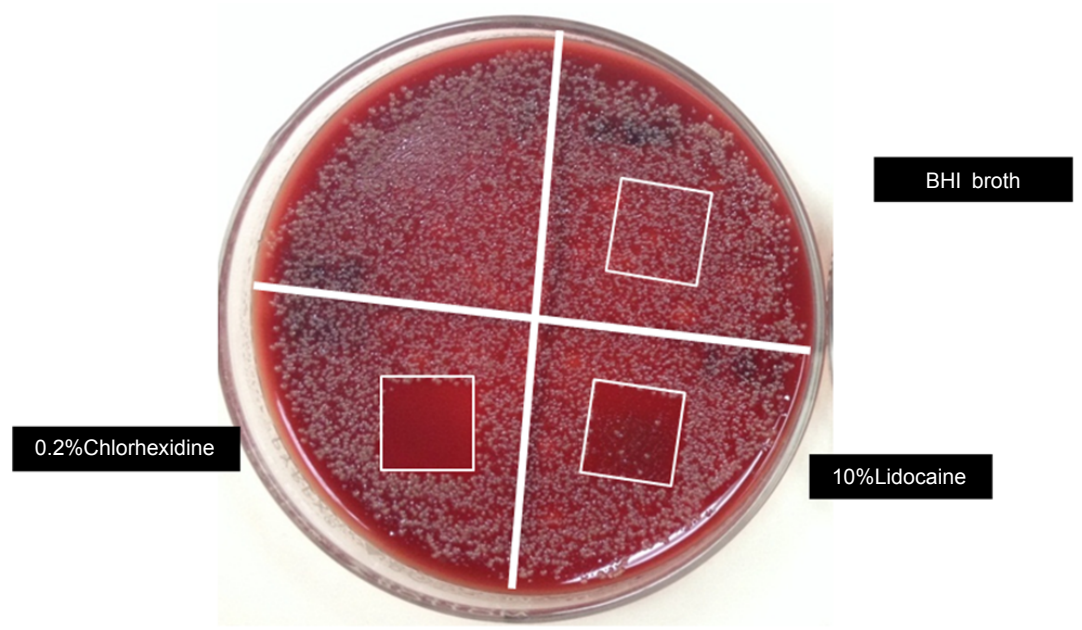

Fig. 2. Representative results of antimicrobial effects of $10 \%$ lidocaine and $0.2 \%$ chlorhexidine against oral microflora collected from a volunteer; exposure time of $3 \mathrm{~min}$.

and $S$. pyogenes even after an exposure time of $3 \mathrm{~min}$. LD was less effective than CHX; within 3 min the growth of E. coli, S. salivarius, and S. viridans was less than $50 \%$ and there was no effect against $S$. aureus, E. faecalis, and $S$. pyogenes.

Regarding oral microflora, the antimicrobial effect of LD on 22 volunteer samples was shown in Fig. 1. The highest antimicrobial score at the exposure time of $1 \mathrm{~min}$ was 2 in 3 volunteers; at 2 min, was 3 in 4 volunteers; and at $3 \mathrm{~min}$, was 3 in 11 volunteers. Antimicrobial effectiveness of $10 \%$ LD showed statistically significant differences among exposure times $(\mathrm{P}<0.05)$. Within 3 min, LD could not completely inhibit oral microflora from any sample. The antimicrobial score of 3 was shown in Fig. 2. In contrast, $0.2 \%$ CHX showed a bactericidal effect against all samples (antimicrobial score of 4), as shown in Fig. 2.

\section{Antimicrobial effectiveness of $10 \%$ LD against the oral microflora on oral mucosa}

Results of antimicrobial effectiveness of $10 \%$ LD when applied on buccal mucosa were shown in Fig. 3. The highest antimicrobial score at the exposure time of $1 \mathrm{~min}$ was 3 in only 1 volunteer; at $2 \mathrm{~min}$, the score was 3 in 4 volunteers; and at $3 \mathrm{~min}$, the score was 3 in 13 volunteers. Statistical analysis indicated the significant 


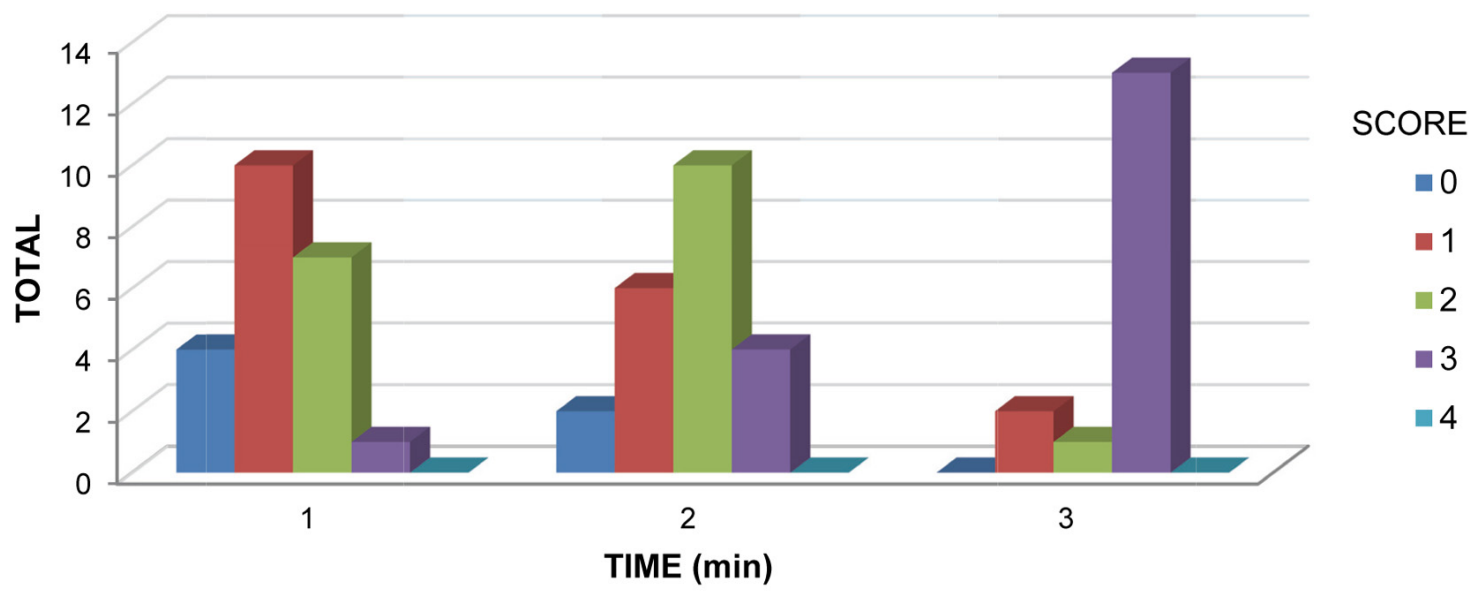

Fig. 3. Antimicrobial effectiveness of $10 \%$ lidocaine against oral microflora from the buccal mucosa of 22 volunteers; exposure time of 1,2 , and 3 min. Antimicrobial score: $4=>95 \%$ bacterial reduction, $3=60-95 \%$ bacterial reduction, $2=40-60 \%$ bacterial reduction, $1=5-40 \%$ bacterial reduction, $0=<5 \%$ bacterial reduction.
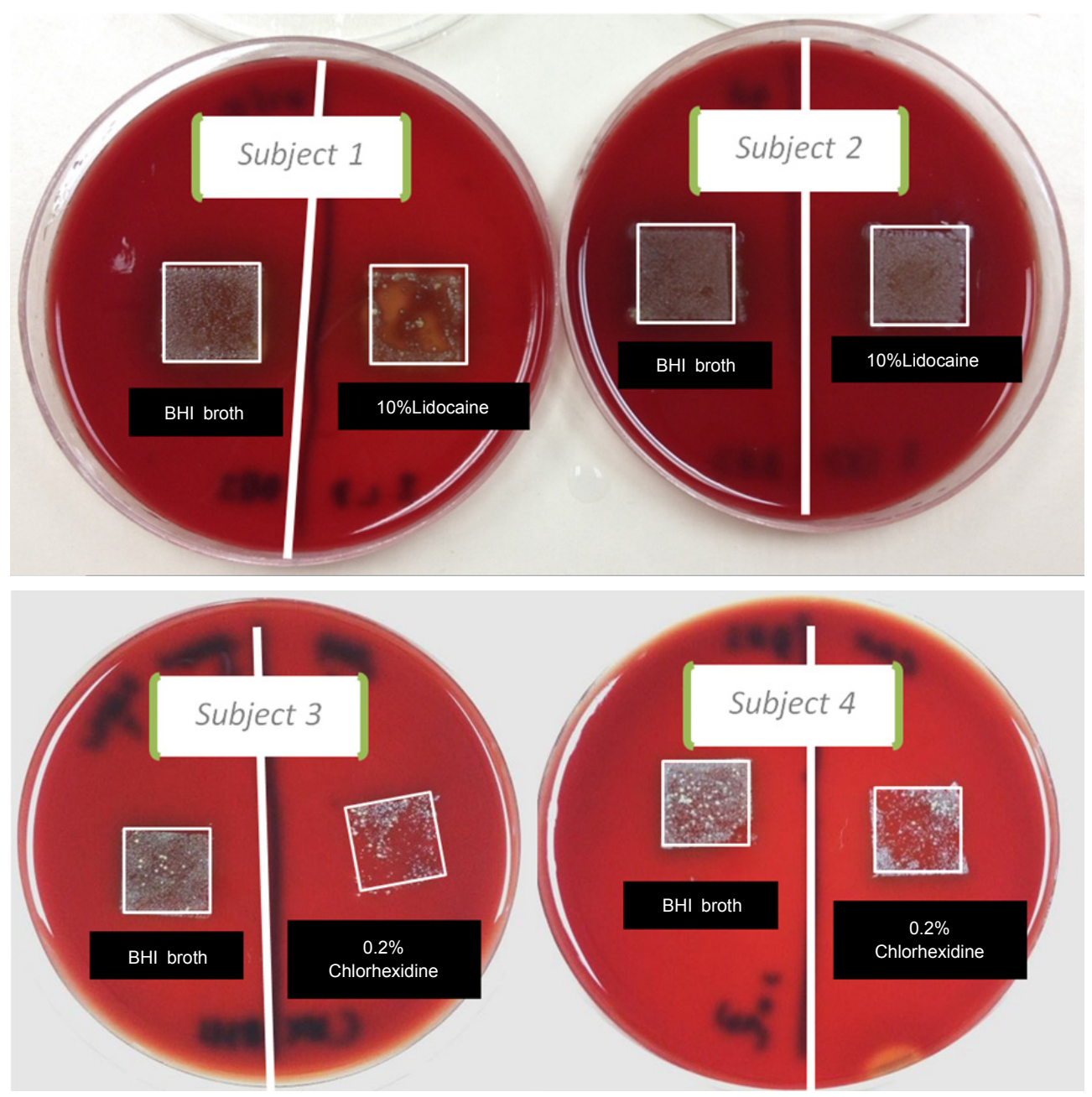

Fig. 4. Representative results of antimicrobial effects of $10 \%$ lidocaine and $0.2 \%$ chlorhexidine against oral microflora from the buccal mucosa of 4 volunteers; exposure time of $3 \mathrm{~min}$. 
differences of antimicrobial effectiveness of $10 \% \mathrm{LD}$ among exposure times $(\mathrm{P}<0.05)$. Within $3 \mathrm{~min}$, both $0.2 \% \mathrm{CHX}$ and $10 \%$ LD could not completely kill oral microflora from any volunteers. The antimicrobial effect against microflora from 4 volunteers was demonstrated in Fig. 4. Subject nos. 1, 2, 3, and 4 showed antimicrobial scores of $2,0,3$, and 1 , respectively.

\section{DISCUSSION}

The administration of local anesthesia in dental procedures by needle injection through the oral mucosa may introduce colonized oral microflora into the blood circulation $[9,16]$. Some local anesthetic injections, i.e., buccal infiltration analgesia, conventional intraligamental analgesia, and modified intraligamental analgesia, can cause bacteremia [14]. Another study has shown increases in bacterial levels in the blood stream immediately after local anesthetic injection and the level were decreased subsequently [17]. Nevertheless, antibiotic prophylaxis is recommended prior to some dental treatment, i.e., tooth extraction, root scaling to reduce bacteremia, particularly in immunocompromised patients or patients receiving immunosuppressive therapy. Previous studies reported that prophylactic penicillin $\mathrm{G}$ prior to tooth extractions and dental cleaning decreased bacteremia rates [18-19].

Several previous studies have shown that local anesthesia also possesses antimicrobial properties. The first observation was made in 1909 and followed by others which studied several anesthetic preparations used in dentistry $[4-6,8,13,20]$. The studies were conducted in vitro using various microbial species, including oral microflora species and opportunistic pathogens. Pelz et al. tested the antimicrobial activity of seven local anesthetics currently used in dentistry, i.e., Ultracaine D-S (articaine hydrochloride), Carbostesin (bupivacaine hydrochloride), Scandicaine (mepivacaine hydrochloride), Xylonest (prilocaine hydrochloride), Xylocaine (LD hydrochloride), Hostacaine (butanilicaine phosphate), and Novacaine (procaine hydrochloride) against a number of commensal and pathogenic species found in the oral cavity [8]. They determined the minimal inhibitory concentration (MIC) and minimal bactericidal concentration (MBC) and found that the MBC values of local anesthetics were lower than the concentration that was routinely used for injection. It suggested that local anesthetics may be able to inhibit microorganisms entering the soft tissue during needle injection.

Topical anesthetics were generally applied on sensitive areas to collect samples or to the injection site in order to block pain. The antimicrobial effect of topical anesthetics in the dental field has been shown to be effective against both oral and non-oral bacteria [4,5,7,9]. Using the agar disc diffusion method, Gocmen et al., compared the antimicrobial effect of $5 \% \mathrm{LD}, 2.5 \% \mathrm{LD} /$ prilocaine, and $2 \%$ mupirocin (an antimicrobial agent) against six bacterial strains: S. aureus, S. epidermidis, S. pyogenes, E. faecalis, Pseudomonas aeruginosa, and E. coli [13]. Both topical local anesthetics were found to be effective against $S$. aureus, S. epidermidis, E. coli, and S. pyogenes, whereas they had no effects on $P$. aeruginosa. Only 5\% LD was effective against E. faecalis. However, the inhibition zones of both anesthetics were smaller than those of mupirocin.

Our in vitro results, in agreement with the previous studies, show differences in susceptibility of selected oral and non-oral bacterial strains to $10 \%$ LD. The antimicrobial effect was increased as the exposure time increased from 1 to $3 \mathrm{~min}$. Parr et al. reported that the killing effect of LD was time and dose dependent; gram-negative bacteria were more susceptible than gram-positive bacteria [15]. In the case of mixed oral microflora from buccal mucosa, a higher number of samples with the highest antimicrobial effect was obtained when the exposure time increased from 1 to 3 $\min$. Nevertheless, the short exposure time of $3 \mathrm{~min}$ was not enough to inhibit all bacteria. We conducted antimicrobial effectiveness in vivo or in other words, evaluated the effect of anesthetic agents against biofilms on oral mucosa at the site of injection which was comprised different species in varying concentrations. We 
mimicked the clinical situation by placing the filter paper soaked with topical $10 \%$ LD spray on the buccal mucosa and determined the antimicrobial effect within the duration of 1-3 min. The imprint technique was used to collect oral biofilms on the buccal mucosa for bacterial culturing.

To our knowledge, this is the first in vivo study which showed the antimicrobial effectiveness of topical local anesthetic spray against mixed microflora of the oral cavity. Our results suggested the 10\% LD topical anesthetic spray can reduce the amount of oral biofilms on the buccal mucosa by $60-95 \%$ when compared with controls without the anesthetic. According to the results from the laboratory study, the effectiveness in each individual is different owing to the species, concentration, and exposure time. The mechanism of action on microbial survival is not well understood. Some researchers suggested that it may be related to an adverse effect on the bacterial cell wall or cell membrane permeability, which is related to the lipophilic nature of the drug or upon the synthesis of cell membrane precursors [21]. Other suggested mechanisms are the dysfunction of cellular respiration, or the alteration of DNA, RNA, and protein synthesis $[20,22]$.

In conclusion, this study demonstrated the antimicrobial activity of $10 \%$ LD topical anesthetic spray in vitro and determined its application on buccal mucosa for 3 min could cause a reduction in oral microflora. The antimicrobial effect of local anesthetic use in dentistry may explain the low incidence of local infections and injection abscesses. The factors which interfered with the effectiveness in vivo are the drug concentration, the dilution by saliva, contact time, the number and type of microorganisms present and the presence of mucus. The suggested use of an antiseptic mouthwash to reduce oral bacteria may enhance the antimicrobial effectiveness of subsequent applications of local anesthetic spray. In addition, the use of antibiotic prophylaxis and antiseptic agents in immunocompromised patients should be considered to prevent infections by opportunistic pathogens.
Acknowledgements: We would like to express our appreciation to the dental students who were volunteers in this study, with special thanks to Ms. Punsiri Vungkahard, Ms. Pichamon Sriviriyakul, Ms. Pimanong Thongsamrith, Ms. Sansanee Neelawatanasook, and Ms. Supitcha Pitaksakul. We wish to thank Mr. Arthit Klaophimai for his assistance in the microbiology laboratory, staff from the Oral and Maxillofacial surgery department for assistance during the clinical procedures. This research was partially supported by the Faculty of Dentistry, Mahidol University. Funding: None

Declaration of interests: None

Ethical Approval: This study was approved by the Committee for Human Research, the faculty of Dentistry and the faculty of Pharmacy at Mahidol University (MU-DT/PY-IRB 2014/DT098).

\section{REFERENCES}

1. Erlich H. Bacteriologic studies and effects of anesthetic solutions on bronchial secretions during bronchoscopy. Am Rev Respirat Doseases 1961; 84: 414-21.

2. Kleinfeld J, Ellis PP. Inhibition of microorganisms by topical anaesthetics. Applied Microbiol 1967; 15: 1296-8.

3. Kleinfeld J, Ellis PP. Effects of topical anesthetics on growth of microorganisms. Arch Opthalmol 1966; 76: $712-5$.

4. Sculley PD, Dunley RE. Antimicrobial activity of a lidocaine preparation. Anesth Prog 1980; 27: 21-3.

5. Morrow ME, Berry CW. Antimicrobial properties of topical anesthetic liquids containing lidocaine or benzocaine. Anesth Prog 1988; 35: 9-15.

6. Feldman JM, Chapin-Robertson K, Turner J. Do agents for epidural analgesia have antimicrobial properties? Reg Anesth 1994; 19: 43-7.

7. Berg JO, Mössner BK, Skov MN, Lauridsen J, Gottrup F, Kolmos HJ. Antibacterial properties of EMLA® and lidocaine in wound tissue biopsies for cultuting. Wound Rep Reg 2006; 14: 581-5. 
8. Pelz K, Wiedmann-Al-Ahmad M, Bogdan C, Otten JE. Analysis of the antimicrobial activity of local anaesthetics used for dental analgesia. JMM 2008; 57: 88-94.

9. Winther JE, Praphailong L. Antimicrobial effect of anesthetic sprays. Acta Odont Scand 1969; 27: 205-18.

10. Mullin GS, Rubinfeld RS. The antibacterial activity of topical anesthetics. Cornea 1997; 16: 662-5.

11. Aydin ON, Eyigor M, Aydin N. Antimicrobial activity of ropivacaine and other local anesthetics. Eur J Anesthesiol 2001; 18: 687-94.

12. Kerenyi M, Batai MR, Juhasz V, Batai I. Lidocaine/ prilocaine ointment (EMLA) has an antibacterial effect in vitro. J Hosp Infect 2004; 56: 75-6.

13. Gocmen JS, Buyukkocak U, Caglayan O. In vitro antibacterial effects of topical local anesthetics. J Dermatolog Treat 2008; 19: 351-353.

14. Robert GJ, Simmons NB, Longhurst P, Hewitt PB. Bacteraemia following local anaesthetic injections in children. Br Dent J 1998; 185: 295-8.

15. Parr AM, Zoutman DE, Davidson JS. Antimicrobial activity of lidicaine against bacteria associated with nosocomial wound infection. Ann Plast Surg 1999; 43: 239-45.

16. Streitfeld MM, Zinner DD. Microbiological hazards of local dental anesthesia. Pilot study of involuntary aspiration of bacteria into hyperdermic needles and anesthetic cartridges after injection. J Am Dent Asso 1958; 57: 657-64.

17. Barbosa M, Carmona IT, Amaral B, Limeres J, Álvarez M, Cerqueira C, et al. General anesthesia increases the risk of bacteremia following dental extractions. Oral Surg Oral Med Oral Pathol Oral Radiol Endod 2010; 110: 706-12.

18. Baltch AL, Pressman HL, Hammer MC, Sutphen NC, Smith RP, Shayegani M. Bacteremia following dental extractions in patients with and without penicillin prophylaxis. Am J Med Sci 1982a; 283: 129-40.

19. Baltch AL, Schaffer C, Hammer MC, Sutphen NC, Smith RP, Shayegani M, et al. Bacteremia following dental cleaning in patients with and without penicillin prophylaxis. Am Heart J 1982; 104: 1335-9.

20. Schmidt RM, Rosenkranz HS. Antimicrobial activity of local anesthetic: lidocaine and procaine. J Infec Dis 1970; 121: 598-607.

21. Silva MT, Sousa JC, PolÒonia JJ, Macedo PM. Effects of local anesthetics on bacterial cells. J Bacteriol 1979; 137: $461-8$.

22. Fazly BB, Salt WG. Local anesthetics as antimicrobial agents: structure-action considerations. Microbs 1983; 37: 45-64. 